\title{
Curvature Estimates in Asymptotically Flat Manifolds of Positive Scalar Curvature
}

\author{
Felix Finster AND Ines Kath
}

We consider an asymptotically flat Riemannian spin manifold of positive scalar curvature. An inequality is derived which bounds the Riemann tensor in terms of the total mass and quantifies in which sense curvature must become small when the total mass tends to zero.

\section{Introduction.}

Suppose that $\left(M^{n}, g\right)$ is an asymptotically flat Riemannian spin manifold of positive scalar curvature. The positive mass theorem $[1,2,3]$ states that the total mass of the manifold is always positive, and is zero if and only if the manifold is flat. This result suggests that there should be an inequality which bounds the Riemann tensor in terms of the total mass and implies that curvature must become small when the total mass tends to zero. In [4] such curvature estimates were derived in the context of General Relativity for 3-manifolds being hypersurfaces in a Lorentzian manifold. In the present paper, we study the problem more generally on a Riemannian manifold of dimension $n \geq 3$. Our curvature estimates then give a quantitative relation between the local geometry and global properties of the manifold.

The main difficulty in higher dimensions is to bound the Weyl tensor (which for $n=3$ vanishes identically). Our basic strategy for controlling the Weyl tensor can be understood from the following simple consideration. The existence of a parallel spinor in an open set $U \subset M$ implies that the manifold is Ricci flat in $U$. Thus it is reasonable that by getting suitable estimates for the derivatives of a spinor, one can bound all components of the Ricci tensor. This method is used in [4], where a solution of the Dirac equation is analyzed using the Weitzenböck formula. But the local existence of a parallel spinor does not imply that the Weyl tensor vanishes. This is the underlying reason why in dimension $n>3$, our estimates cannot be obtained by looking at one spinor, but we must consider a family $\left(\psi^{i}\right)_{i=1, \ldots, 2^{[n / 2]}}$ of solutions of the Dirac equation. Out of these solutions we form the so-called spinor operator $P_{x}$. The curvature tensor can be bounded in terms of suitable derivatives 
of $P_{x}$, and an integration-by-parts argument, the Weitzenböck formula, and a-priori estimates for the spinor operator give the desired result.

We now give the precise statement of our result. For simplicity, we consider only one asymptotically flat end. The following definition immediately generalizes that used in [4]; for a slightly more general definition see [3].

Definition 1.1. A Riemannian manifold $\left(M^{n}, g\right), n \geq 3$, is said to be asymptotically flat if there is a compact set $K \subset M$ and a diffeomorphism $\Phi$ which maps $M \backslash K$ to the region $\mathbb{R}^{n} \backslash B_{r_{0}}(0)$ outside a ball of radius $r_{0}$. Under this diffeomorphism, the metric should be of the form

$$
\left(\Phi_{*} g\right)_{i j}=\delta_{i j}+\mathcal{O}\left(r^{2-n}\right), \quad \partial_{k}\left(\Phi_{*} g\right)_{i j}=\mathcal{O}\left(r^{1-n}\right), \quad \partial_{k l}\left(\Phi_{*} g\right)_{i j}=\mathcal{O}\left(r^{-n}\right) .
$$

Furthermore, scalar curvature should be in $L^{1}(M)$.

For an asymptotically flat manifold the total mass $m$ is defined by

$$
m=\frac{1}{c(n)} \lim _{\rho \rightarrow \infty} \int_{S_{\rho}}\left(\partial_{j}\left(\Phi_{*} g\right)_{i j}-\partial_{i}\left(\Phi_{*} g\right)_{j j}\right) d \Omega^{i},
$$

where $c(n)>0$ is a normalization constant (which can be chosen arbitrarily), $d \Omega$ is the volume form on the sphere $S_{\rho} \subset \mathbb{R}^{n}$ of radius $\rho$, and $d \Omega^{i}$ denotes the product of $d \Omega$ by the i-th component of the normal vector of $S_{\rho}$. As shown in [3], this definition is independent of the choice of $\Phi$. For our estimates, we also need the isoperimetric constant $k$ given by

$$
k=\inf \frac{A}{V^{\frac{n-1}{n}}},
$$

where the infimum is taken over all smooth regions $D, V$ is the $n$-volume of $D$, and $A$ is the $(n-1)$-volume of the boundary of $D$.

Theorem 1.2. Let $M^{n}$ be an asymptotically flat Riemannian spin manifold of positive scalar curvature. Then there are positive constants $c_{1}, c_{2}$, and $c_{3}$ depending only on $n$ as well as a set $D$ with

$$
\mu(D) \leq\left(\frac{c_{3} m}{k^{2}}\right)^{\frac{n}{n-2}}
$$

such that for all positive $\eta \in C^{\infty}(M)$ with $\sup _{M}(|\eta|+|\nabla \eta|)<\infty$ the following inequality holds,

$$
\int_{M \backslash D} \eta|R|^{2} d \mu \leq m c_{1} \sup _{M}(|\eta R|+|\Delta \eta|)+\sqrt{m} c_{2}\|\eta \nabla R\|_{2} .
$$

Here $R$ is the Riemann tensor, $m$ the total mass, and $k$ the isoperimetric constant, $\|\cdot\|_{2}$ denotes the $L^{2}$-Norm on $M$. 
One application of the above theorem is to a continuous family $\left(M_{\lambda}\right)_{\lambda \geq 0}$ of manifolds (e.g. obtained by a flow of the metric). If $\lim _{\lambda \rightarrow \infty} m(\lambda)=0$, $\sup _{M}|R|$ and $\|\nabla R\|_{2}$ are uniformly bounded, and the isoperimetric constant is bounded away from zero, then our theorem implies that the Riemann tensor converges to zero pointwise almost everywhere, and thus the manifold becomes flat. For other applications see [4].

\section{Spinors, the Dirac Equation.}

In this section we recall some basic facts concerning spinors and the Dirac equation; for details see $[6,7,3]$. Let $\left(M^{n}, g\right)$ be an $n$-dimensional (oriented) Riemannian spin manifold with spin structure $Q$ and spinor bundle

$$
S=Q \times_{\operatorname{Spin}(n)} \Delta_{n},
$$

which is associated with $Q$ by the spinor representation $\Delta_{n}$. As a vector space, $\Delta_{n}$ is equal to $\mathbb{C}^{N}, N:=2^{[n / 2]}$. The canonical Hermitian product on $\Delta_{n}$ defines a complex scalar product $\langle\cdot, \cdot\rangle$ on $S$. We denote the real part of this scalar product by $(\cdot, \cdot)$. The Levi-Civita connection $\nabla$ on $\left(M^{n}, g\right)$ induces a covariant derivative in $S$ called spinor derivative, which we denote again by $\nabla$. The spinor derivative is compatible with $\langle\cdot, \cdot\rangle$, i.e.

$$
X\langle\varphi, \psi\rangle=\left\langle\nabla_{X} \varphi, \psi\right\rangle+\left\langle\varphi, \nabla_{X} \psi\right\rangle
$$

for all sections $\varphi, \psi$ in $S$ and all vector fields $X$ on $M$. Its curvature tensor

$$
R^{S}(X, Y)=\nabla_{X} \nabla_{Y}-\nabla_{Y} \nabla_{X}-\nabla_{[X, Y]}
$$

is given locally by

$$
R^{S}(X, Y) \psi=\frac{1}{4} \sum_{\alpha, \beta=1}^{n} R\left(X, Y, s_{\alpha}, s_{\beta}\right) s_{\alpha} \cdot s_{\beta} \cdot \psi,
$$

where $s_{1}, \ldots, s_{n}$ is a local orthonormal frame on $\left(M^{n}, g\right), R$ is the Riemannian curvature tensor of $\left(M^{n}, g\right)$ and "." denotes Clifford multiplication of a spinor by a vector. The Clifford multiplication satisfies the anti-commutation relations

$$
X \cdot Y \cdot \psi+Y \cdot X \cdot \psi=-2 g(X, Y) \psi
$$

and is anti-Hermitian,

$$
\langle X \cdot \varphi, \psi\rangle+\langle\varphi, X \cdot \psi\rangle=0 .
$$


The Dirac operator $D$ on $\left(M^{n}, g\right)$ is the composition of the spinor derivative $\nabla: \Gamma(S) \longrightarrow \Gamma(T M \otimes S)$ and the Clifford multiplication, i.e. locally

$$
D=\sum_{\alpha=1}^{n} s_{\alpha} \cdot \nabla_{s_{\alpha}}
$$

The square $D^{2}$ of the Dirac operator satisfies the Weitzenböck formula

$$
D^{2}=\Delta^{S}+\frac{\tau}{4}
$$

where $\Delta^{S}=-\sum_{\alpha=1}^{n}\left(\nabla_{s_{\alpha}} \nabla_{s_{\alpha}}+\left(\operatorname{div} s_{\alpha}\right) \nabla_{s_{\alpha}}\right)$ denotes the Bochner-Laplace operator with respect to the spinor derivative and $\tau$ is the scalar curvature of $\left(M^{n}, g\right)$.

Now assume furthermore that $M$ is asymptotically flat and has positive scalar curvature. In the coordinates induced by the diffeomorphism $\Phi$ of Definition 1.1, we choose a constant spinor $\psi_{0}$ and consider the boundary problem

$$
D \psi=0, \quad \lim _{|x| \rightarrow \infty} \psi(x)=\psi_{0} .
$$

It is shown in [3] that this boundary problem has a unique weak solution $\psi$. Using the asymptotic form of the metric in Definition 1.1 and elliptic regularity theory, it follows that $\psi$ is even smooth and decays at infinity like

$$
\psi=\psi_{0}+\mathcal{O}\left(r^{2-n}\right), \quad \partial_{k} \psi=\mathcal{O}\left(r^{1-n}\right), \quad \partial_{k l} \psi=\mathcal{O}\left(r^{-n}\right) .
$$

This solution of the Dirac equation can be used to prove the positive mass theorem $[2,3]$, as we now briefly outline. Consider the vector field

$$
X=\frac{1}{2} \operatorname{grad}|\psi|^{2} .
$$

Using the Weitzenböck formula and the positivity of scalar curvature, we can estimate the divergence of $X$ as follows,

$$
\begin{aligned}
\operatorname{div} X & =\sum_{\alpha=1}^{n} s_{\alpha}\left(\nabla_{s_{\alpha}} \psi, \psi\right)=-\left(\Delta^{S} \psi, \psi\right)+|\nabla \psi|^{2} \\
& =\frac{\tau}{4}|\psi|^{2}+|\nabla \psi|^{2} \geq|\nabla \psi|^{2} .
\end{aligned}
$$

We introduce the balls $B_{\rho}$ and spheres $S_{\rho}$ of radius $\rho>r_{0}$ by

$$
D_{\rho}=K \cup \Phi^{-1}\left(B_{\rho}(0) \backslash B_{r_{0}}(0)\right), \quad S_{\rho}=\partial D_{\rho}
$$


with $\Phi, r_{0}$, and $K$ as in Definition 1.1, $\rho>r_{0}$, and denote the outer normal on $S_{\rho}$ by $\nu$. We integrate over $D_{\rho}$ and apply Gauss' theorem,

$$
\int_{S_{\rho}} g(X, \nu) d S_{\rho}=\int_{D_{\rho}} \operatorname{div} X d \mu \geq \int_{D_{\rho}}|\nabla \psi|^{2} d \mu .
$$

An asymptotic expansion near infinity [3] shows that as $\rho \rightarrow \infty$, the left side of (2.10) can up to a constant be identified with the boundary integral in (1.1); more precisely,

$$
\lim _{\rho \rightarrow \infty} 4 \int_{S_{\rho}} g(X, \nu) d S \rho=c(n)\left|\psi_{0}\right|^{2} m .
$$

Hence in (2.10) we can take the limit $\rho \rightarrow \infty$ to obtain

$$
c(n)\left|\psi_{0}\right|^{2} m \geq 4\|\nabla \psi\|_{2}^{2} .
$$

This inequality shows that $m \geq 0$. If $m=0,(2.11)$ yields that for any $\psi_{0}$, there is a parallel spinor with $\lim _{|x| \rightarrow \infty} \psi(x)=\psi_{0}$, and this implies that the manifold is flat.

The inequality (2.8) immediately gives an a-priori bound for the spinor, which will be very useful later on. Namely, (2.7) and (2.8) imply that

$$
\Delta|\psi|^{2}=-2 \operatorname{div} X \leq 0 .
$$

Thus $|\psi|^{2}$ is sub-harmonic, and the maximum principle yields that for every solution of the boundary problem (2.5),

$$
\sup _{M}|\psi| \leq\left|\psi_{0}\right| \text {. }
$$

\section{The Integration-by-Parts Argument.}

In this section we derive an $L^{2}$-bound for the second derivative of a solution of the Dirac equation (2.5). The argument is similar to that in dimension three [4]. We give it in some detail, using the formalism of orthonormal frames [6].

We define a vector field $Y$ on $M$ by

$$
Y=\frac{1}{2} \operatorname{grad}|\nabla \psi|^{2} .
$$


Lemma 3.1. For any local orthonormal frame $s_{1}, \ldots, s_{n}$ on $M$,

$$
\begin{gathered}
\operatorname{div} Y=-\left(\nabla \Delta^{S} \psi, \nabla \psi\right)+\left|\nabla^{2} \psi\right|^{2} \\
+\sum_{\alpha, \beta=1}^{n}\left(2\left(R^{S}\left(s_{\alpha}, s_{\beta}\right) \nabla_{s_{\alpha}} \psi, \nabla_{s_{\beta}} \psi\right)+\left(\left(\nabla_{s_{\alpha}} R^{S}\right)\left(s_{\alpha}, s_{\beta}\right) \psi, \nabla_{s_{\beta}} \psi\right)\right. \\
\left.\quad+\operatorname{Ric}\left(s_{\alpha}, s_{\beta}\right)\left(\nabla_{s_{\alpha}} \psi, \nabla_{s_{\beta}} \psi\right)\right) .
\end{gathered}
$$

Proof: With respect to $s_{1}, \ldots, s_{n}$, the vector field $Y$ is

$$
Y=\frac{1}{2} \sum_{\alpha, \beta=1}^{n} \nabla_{s_{\alpha}}\left\langle\nabla_{s_{\beta}} \psi, \nabla_{s_{\beta}} \psi\right\rangle s_{\alpha}=\sum_{\alpha, \beta=1}^{n}\left(\nabla_{s_{\alpha}, s_{\beta}}^{2} \psi, \nabla_{s_{\beta}} \psi\right) s_{\alpha} .
$$

Let $x$ be a point in $M$. For simplicity we choose $s_{1}, \ldots, s_{n}$ such that $\nabla s_{\alpha}=0$ in $x$ for all $\alpha=1, \ldots, n$. Then at $x$,

$$
\begin{aligned}
& \operatorname{div} Y \\
& =\sum_{\alpha, \beta}^{n} s_{\alpha}\left(\nabla_{s_{\alpha}, s_{\beta}}^{2} \psi, \nabla_{s_{\beta}} \psi\right) \\
& =\sum_{\alpha, \beta}^{n}\left(\left(\nabla_{s_{\alpha}} \nabla_{s_{\alpha}} \nabla_{s_{\beta}} \psi, \nabla_{s_{\beta}} \psi\right)-\left(\nabla_{s_{\alpha}} \nabla_{\nabla_{s_{\alpha}} s_{\beta}} \psi, \nabla_{s_{\beta}} \psi\right)\right. \\
& \left.\quad+\left(\nabla_{s_{\alpha}} \nabla_{s_{\beta}} \psi, \nabla_{s_{\alpha}} \nabla_{s_{\beta}} \psi\right)\right) \\
& =\sum_{\alpha, \beta}^{n}\left(\left(\nabla_{s_{\alpha}}\left(\nabla_{s_{\beta}} \nabla_{s_{\alpha}} \psi+\nabla_{\left[s_{\alpha}, s_{\beta}\right]} \psi+R^{S}\left(s_{\alpha}, s_{\beta}\right) \psi\right), \nabla_{s_{\beta}} \psi\right)\right. \\
& \left.\quad-\left(\nabla_{s_{\alpha}} \nabla_{\nabla_{s_{\alpha}} s_{\beta}} \psi, \nabla_{s_{\beta}} \psi\right)+\left(\nabla_{s_{\alpha}} \nabla_{s_{\beta}} \psi, \nabla_{s_{\alpha}} \nabla_{s_{\beta}} \psi\right)\right) \\
& =\sum_{\alpha, \beta}^{n}\left(\left(\nabla_{s_{\beta}} \nabla_{s_{\alpha}} \nabla_{s_{\alpha}} \psi+R^{S}\left(s_{\alpha}, s_{\beta}\right) \nabla_{s_{\alpha}} \psi+\nabla_{s_{\alpha}} \nabla_{\left[s_{\alpha}, s_{\beta}\right]} \psi\right.\right. \\
& \left.\quad+\left(\nabla_{s_{\alpha}} R^{S}\right)\left(s_{\alpha}, s_{\beta}\right) \psi+R^{S}\left(s_{\alpha}, s_{\beta}\right) \nabla_{s_{\alpha}} \psi-\nabla_{s_{\alpha}} \nabla_{\nabla_{s_{\alpha}} s_{\beta}} \psi, \nabla_{s_{\beta}} \psi\right) \\
& \left.\quad+\left(\nabla_{s_{\alpha}, s_{\beta}}^{2} \psi, \nabla_{s_{\alpha}, s_{\beta}}^{2} \psi\right)\right) \\
& =\sum_{\beta=1}^{n}\left(-\nabla_{s_{\beta}} \Delta^{S} \psi, \nabla_{s_{\beta}} \psi\right) \\
& \quad+\sum_{\alpha, \beta}^{n}\left(\left(-\nabla_{s_{\beta}}\left(\operatorname{div} s_{\alpha} \cdot \nabla_{s_{\alpha}} \psi\right)+2 R^{S}\left(s_{\alpha}, s_{\beta}\right) \nabla_{s_{\alpha}} \psi\right.\right. \\
& \left.\left.\quad-\nabla_{s_{\alpha}} \nabla_{\nabla_{s_{\beta}} s_{\alpha}} \psi+\left(\nabla_{s_{\alpha}} R^{S}\right)\left(s_{\alpha}, s_{\beta}\right) \psi, \nabla_{s_{\beta}} \psi\right)+\left(\nabla_{s_{\alpha}, s_{\beta}}^{2} \psi, \nabla_{s_{\alpha}, s_{\beta}}^{2} \psi\right)\right) .
\end{aligned}
$$


Therefore it remains to show that at $x$,

(3.1) $\sum_{\alpha=1}^{n}\left(-\nabla_{s_{\beta}}\left(\operatorname{div} s_{\alpha} \cdot \nabla_{s_{\alpha}} \psi\right)-\nabla_{s_{\alpha}} \nabla_{\nabla_{s_{\beta}} s_{\alpha}} \psi\right)=\sum_{\alpha=1}^{n} \operatorname{Ric}\left(s_{\alpha}, s_{\beta}\right) \nabla_{s_{\alpha}} \psi$.

Since the $s_{\alpha}$ are orthonormal,

$-s_{\beta}\left(\operatorname{div} s_{\alpha}\right)$

$=-s_{\beta}\left(\sum_{\gamma=1}^{n} g\left(\nabla_{s_{\gamma}} s_{\alpha}, s_{\gamma}\right)\right)=s_{\beta}\left(\sum_{\gamma=1}^{n} g\left(s_{\alpha}, \nabla_{s_{\gamma}} s_{\gamma}\right)\right)=\sum_{\gamma=1}^{n} g\left(s_{\alpha}, \nabla_{s_{\beta}} \nabla_{s_{\gamma}} s_{\gamma}\right)$,

and thus

$-\sum_{\alpha=1}^{n} \nabla_{s_{\beta}}\left(\operatorname{div} s_{\alpha} \cdot \nabla_{s_{\alpha}} \psi\right)=\sum_{\alpha, \gamma=1}^{n} g\left(s_{\alpha}, \nabla_{s_{\beta}} \nabla_{s_{\gamma}} s_{\gamma}\right) \nabla_{s_{\alpha}} \psi=\sum_{\beta, \gamma=1}^{n} \nabla_{\nabla_{s_{\beta}} \nabla_{s_{\gamma}} s_{\gamma}} \psi$.

On the other hand, using that $\nabla_{s_{\beta}} s_{\alpha}(x)=0$,

$$
\begin{aligned}
\nabla_{s_{\alpha}} \nabla_{\nabla_{s_{\beta}} s_{\alpha}} \psi & =\nabla_{\nabla_{s_{\beta}} s_{\alpha}} \nabla_{s_{\alpha}} \psi+R^{S}\left(s_{\alpha}, \nabla_{s_{\beta}} s_{\alpha}\right) \psi+\nabla_{\left[s_{\alpha}, \nabla_{s_{\beta}} s_{\alpha}\right]} \psi \\
& =\nabla_{\left[s_{\alpha}, \nabla_{s_{\beta}} s_{\alpha}\right]} \psi=\nabla_{\nabla_{s_{\alpha}} \nabla_{s_{\beta}} s_{\alpha}} \psi
\end{aligned}
$$

Hence at $x$,

$$
\begin{aligned}
& \sum_{\alpha=1}^{n}\left(-\nabla_{s_{\beta}}\left(\operatorname{div} s_{\alpha} \cdot \nabla_{s_{\alpha}} \psi\right)-\nabla_{s_{\alpha}} \nabla_{\nabla_{s_{\beta}} s_{\alpha}} \psi\right) \\
& =\sum_{\alpha=1}^{n}\left(\nabla_{\nabla_{s_{\beta}} \nabla_{s_{\alpha}} s_{\alpha}} \psi-\nabla_{\nabla_{s_{\alpha}} \nabla_{s_{\beta}} s_{\alpha}} \psi\right)=\sum_{\alpha=1}^{n} \nabla_{R\left(s_{\beta}, s_{\alpha}\right) s_{\alpha}} \psi \\
& =\sum_{\alpha=1}^{n} \operatorname{Ric}\left(s_{\alpha}, s_{\beta}\right) \nabla_{s_{\alpha}} \psi
\end{aligned}
$$

Corollary 3.2. For $\psi$ a solution of (2.5) with $\left|\psi_{0}\right|=1$ and $\eta$ a positive smooth function with $\sup _{M}(|\eta|+|\nabla \eta|)<\infty$,

$$
\int_{M} \eta\left|\nabla^{2} \psi\right|^{2} d \mu \leq m C_{1}(n) \sup _{M}(|\eta R|+|\Delta \eta|)+\sqrt{m} C_{2}(n)\|\eta \nabla R\|_{2} .
$$


Proof: We multiply the result of Lemma 3.1 by $\eta$ and integrate over the ball $D_{\rho},(2.9)$. Using Gauss' theorem and the definition of $Y$,

$$
\begin{gathered}
\int_{D_{\rho}} \eta\left|\nabla^{2} \psi\right|^{2} d \mu=\int_{S_{\rho}}\left(\eta g(Y, \nu)-\frac{1}{2}|\nabla \psi|^{2} g(\nabla \eta, \nu)\right) d S_{\rho} \\
-\frac{1}{2} \int_{D_{\rho}}|\nabla \psi|^{2} \cdot \Delta \eta d \mu+\int_{D_{\rho}} \eta\left(\nabla \Delta^{S} \psi, \nabla \psi\right) d \mu \\
-\int_{D_{\rho}} \eta \sum_{\alpha, \beta=1}^{n}\left(2\left(R^{S}\left(s_{\alpha}, s_{\beta}\right) \nabla_{s_{\alpha}} \psi, \nabla_{s_{\beta}} \psi\right)+\left(\left(\nabla_{s_{\alpha}} R^{S}\right)\left(s_{\alpha}, s_{\beta}\right) \psi, \nabla_{s_{\beta}} \psi\right)\right. \\
\left.+\operatorname{Ric}\left(s_{\alpha}, s_{\beta}\right)\left(\nabla_{s_{\alpha}} \psi, \nabla_{s_{\beta}} \psi\right)\right) d \mu
\end{gathered}
$$

Taking the limit $\rho \rightarrow \infty$, the integral over $S_{\rho}$ tends to zero because of the asymptotic behavior of $\psi(2.6)$. Furthermore, we can estimate the remaining terms in (3.2) according to

$$
\begin{aligned}
& \sum_{\alpha, \beta=1}^{n}\left|2\left(R^{S}\left(s_{\alpha}, s_{\beta}\right) \nabla_{s_{\alpha}} \psi, \nabla_{s_{\beta}} \psi\right)\right| \\
& \left.=\frac{1}{2} \sum_{\alpha, \beta, \gamma, \delta=1}^{n} \mid R\left(s_{\alpha}, s_{\beta}, s_{\gamma}, s_{\delta}\right) s_{\gamma} \cdot s_{\delta} \cdot \nabla_{s_{\alpha}} \psi, \nabla_{s_{\beta}} \psi\right)\left.\left|\leq \tilde{c}_{1}(n)\right| R|| \nabla \psi\right|^{2} \\
& \quad \sum_{\alpha, \beta=1}^{n}\left|\left(\left(\nabla_{s_{\alpha}} R^{S}\right)\left(s_{\alpha}, s_{\beta}\right) \psi, \nabla_{s_{\beta}} \psi\right)\right| \\
& =\frac{1}{4} \sum_{\alpha, \beta, \gamma, \delta=1}^{n}\left|\left(\left(\nabla_{s_{\alpha}} R\right)\left(s_{\alpha}, s_{\beta}, s_{\gamma}, s_{\delta}\right) s_{\gamma} \cdot s_{\delta} \cdot \psi, \nabla_{s_{\beta}} \psi\right)\right| \\
& \leq \tilde{c}_{2}(n)|\nabla R||\psi||\nabla \psi| \\
& \sum_{\alpha, \beta=1}^{n} \operatorname{Ric}\left(s_{\alpha}, s_{\beta}\right)\left(\nabla_{s_{\alpha}} \psi, \nabla_{s_{\beta}} \psi\right) \leq \tilde{c}_{3}(n)|R||\nabla \psi|^{2} \\
& \left|\left(\nabla \Delta^{S} \psi, \nabla \psi\right)\right|=\sum_{\alpha=1}^{n}\left(\nabla_{s_{\alpha}}\left(\frac{\tau}{4} \psi\right), \nabla_{s_{\alpha}} \psi\right) \mid \\
& \leq \tilde{c}_{4}(n)|\nabla R||\psi||\nabla \psi|+\tilde{c}_{5}(n)|R||\nabla \psi|^{2}
\end{aligned}
$$


with suitable constants $\tilde{c}_{1}, \ldots, \tilde{c}_{4}$. We conclude that

$$
\begin{aligned}
& \int_{D_{r}} \eta\left|\nabla^{2} \psi\right|^{2} d D_{r} \\
& \leq \frac{1}{2} \int_{D_{r}}\left|\nabla^{2} \psi\right|^{2}|\Delta \eta| d D_{r} \\
& \quad+\tilde{C}_{1}(n) \int_{D_{r}}|\eta R||\nabla \psi|^{2} d D_{r}+\tilde{C}_{2}(n) \int_{D_{r}} \eta|\nabla R||\psi||\nabla \psi| d D_{r} \\
& \leq \frac{1}{2}\|\nabla \psi\|_{2}^{2} \sup _{M}|\Delta \eta| \\
& \quad+\tilde{C}_{1}(n)\|\nabla \psi\|_{2}^{2} \sup _{M}|\eta R|+\tilde{C}_{2}(n)\|\eta \nabla R\|_{2}\|\nabla \psi\|_{2} \sup _{M}|\psi|,
\end{aligned}
$$

and the assertion follows from (2.11) and (2.12).

\section{A-Priori Estimates for the Spinor Operator.}

We choose an orthonormal basis of constant spinors $\left(\psi_{0}^{i}\right)_{i=1, \ldots, N}, N=2^{[n / 2]}$, $\left\langle\psi_{0}^{i}, \psi_{0}^{j}\right\rangle=\delta^{i j}$, and denote the corresponding solutions of the boundary problem (2.5) by $\left(\psi^{i}\right)_{i=1, \ldots, N}$. We define the spinor operator $P_{x}$ by

$$
P_{x}: S_{x} M \longrightarrow S_{x} M: \psi \longmapsto \sum_{i=1}^{N}\left\langle\psi_{x}^{i}, \psi\right\rangle \psi_{x}^{i} .
$$

Since at infinity the $\psi^{i}$ go over to an orthonormal basis,

$$
\lim _{|x| \rightarrow \infty} P_{x}=\mathbb{1} .
$$

This section is concerned with a-priori estimates for the operator $P_{x}$. In the following lemma, we use the maximum principle to derive upper bounds for $\left|P_{x}\right|$, where $|$.$| denotes the sup-norm.$

Lemma 4.1. $\left|P_{x}\right| \leq 1$ for all $x \in M$.

Proof: We define the matrix $H$ by

$$
H=\left(h_{i j}\right)_{i=1, \ldots, N} j=1, \ldots, N \quad \text { with } \quad h_{i j}=\left\langle\psi_{x}^{i}, \psi_{x}^{j}\right\rangle .
$$

By definition, $H$ is Hermitian and all eigenvalues of $H$ are real and nonnegative. Consider the spinor $\psi=\sum_{i=1}^{N} a_{i} \psi^{i}$ with $a=\left(a_{1}, \ldots, a_{N}\right)^{\top} \in \mathbb{C}^{N}$. 
Then $\psi$ is a solution of the boundary problem (2.5) with $\psi_{0}=\sum_{i=1}^{N} a_{i} \psi_{0}^{i}$. By (2.12),

$$
\sum_{i, j=1}^{N} \overline{a_{i}} a_{j} h_{i j}=\left|\psi_{x}\right|^{2} \leq\left|\psi_{0}\right|^{2}=\sum_{i=1}^{N}\left|a_{i}\right|^{2}
$$

for all $x \in M$. Since $a$ is arbitrary, all eigenvalues of $H$ must be $\leq 1$. Let $\phi$ be an arbitrary spinor at $x$ and $\psi$ its orthogonal projection to $\operatorname{span}\left\{\psi^{i} \mid i=1, \ldots, N\right\}$. Then $\psi=\sum_{i=1}^{N} a_{i} \psi^{i}$ and

$$
\begin{aligned}
\left|P_{x} \phi\right|^{2} & =\sum_{i, j=1}^{N}\left\langle\psi, \psi_{x}^{i}\right\rangle\left\langle\psi_{x}^{i}, \psi_{x}^{j}\right\rangle\left\langle\psi_{x}^{j}, \psi\right\rangle=\overline{(H a)}^{\top} H(H a) \\
& \leq \bar{a}^{\top} H a=\left|\psi_{x}\right|^{2}=\left|\phi_{x}\right|^{2},
\end{aligned}
$$

where the first inequality uses that there is an orthonormal basis of $\mathbb{C}^{N}$ of eigenvectors of $H$ and that all eigenvalues of $H$ are non-negative and $\leq 1$.

We next derive Sobolev estimates for the Hilbert-Schmidt norm $\|\cdot\|$ of the operator $\mathbb{1}-P_{x}$.

Lemma 4.2. There is a constant $c$ depending only on the dimension $n$ such that for any $\varepsilon>0$,

$$
\left\|\mathbb{1}-P_{x}\right\|^{2}<\varepsilon
$$

except on a set $D(\varepsilon)$ with

$$
\mu(D) \leq\left(\frac{c m}{\varepsilon^{2} k^{2}}\right)^{\frac{n}{n-2}} .
$$

Proof: We set $h(x)=\left\|\mathbb{1}-P_{x}\right\|^{2}$. Choosing an orthonormal basis $\left(\phi^{j}\right)_{j=1, \ldots, N}$ of $S_{x} M$, the trace of $S_{x}$ is computed as follows,

$$
\operatorname{Tr}\left(P_{x}\right)=\sum_{j=1}^{N}\left\langle\phi^{j}, P_{x} \phi^{j}\right\rangle \stackrel{(4.1)}{=} \sum_{i, j=1}^{N}\left\langle\psi^{i}, \phi^{j}\right\rangle\left\langle\phi^{j}, \psi^{i}\right\rangle=\sum_{i=1}^{N}\left\langle\psi^{i}, \psi^{i}\right\rangle .
$$

Similarly we obtain for $h$,

$$
h(x)=\operatorname{Tr}\left(\mathbb{1}-2 P_{x}+P_{x}^{2}\right)=N-2 \sum_{i=1}^{N}\left\langle\psi_{x}^{i}, \psi_{x}^{i}\right\rangle+\sum_{i, j=1}^{N}\left|\left\langle\psi_{x}^{i}, \psi_{x}^{j}\right\rangle\right|^{2},
$$


and the gradient of $h$ is computed to be

$$
\nabla h=-4 \sum_{i=1}^{N} \sum_{\alpha=1}^{n}\left\langle\nabla_{s_{\alpha}} \psi^{i}, \psi^{i}\right\rangle s_{\alpha}+4 \sum_{i=1}^{N} \sum_{\alpha=1}^{n}\left\langle\nabla_{s_{\alpha}} \psi^{i}, P_{x} \psi^{i}\right\rangle s_{\alpha} .
$$

The Schwarz inequality combined with Lemma 4.1 yield that

$$
|\nabla h| \leq 8 n \sum_{i=1}^{N}\left|\nabla \psi^{i}\right|
$$

and applying Hölder's inequality,

$$
|\nabla h|^{2} \leq 64 n^{2} N \sum_{i=1}^{N}\left|\nabla \psi^{i}\right|^{2}
$$

We now integrate over $M$ and substitute in (2.11) to obtain

$$
\|\nabla h\|_{2}^{2} \leq 16 n^{2} N^{2} c(n) m .
$$

The Sobolev inequality yields for some constant $C(n)$,

$$
k^{2}\|h\|_{q}^{2} \leq C\|\nabla h\|_{2}^{2} \quad \text { with } \quad q=\frac{2 n}{n-2},
$$

where $k$ is the isoperimetric constant of $M$ (see Lemma 4.3 for the derivation of this inequality). Hence $h<\varepsilon$ except on a set $D$ of small measure (4.3).

For the reader not familiar with Sobolev inequalitities in non-compact Riemannian manifolds we now give the proof of inequality (4.4).

Lemma 4.3. Let $0 \leq h \in C^{\infty}(M)$ with $\lim _{|x| \rightarrow \infty} h(x)=0$ and $q=2 n /(n-$ 2). Then

$$
\|h\|_{q} \leq \frac{q}{k}\|\nabla h\|_{2}
$$

Proof: We define for $u>0$ the sets

$$
N_{u}=\{x \in M \mid h(x) \geq u\} \quad, \quad S_{u}=\partial N_{u}=h^{-1}(u) .
$$

Since $\lim _{|x| \rightarrow \infty} h(x)=0$, these sets are compact. Sard's lemma yields that, with the exception of $u$ in a set of measure zero, $\nabla h$ does not vanish on 
$S_{u}$, and so $S_{u}$ is a compact submanifold of $M$ of codimension one. We denote the volume of $N_{u}$ by $V_{u}$ and the area of $S_{u}$ by $A_{u}$. Also, $d S_{u}$ denotes the measure on $S_{u}$ corresponding to the induced Riemannian metric. The co-area formula yields that for any $p>0$,

$$
\int_{M} h^{p}|\nabla h| d \mu=\int_{0}^{\infty} d u \int_{S_{u}} h^{p} d S_{u}=\int_{0}^{\infty} u^{p} A_{u} d u .
$$

Furthermore, we have the following estimates,

$$
\begin{aligned}
V_{u} & =\int_{N_{u}} d \mu \leq \frac{1}{u^{q}} \int_{N_{u}} h^{q} d \mu \leq u^{-q}\|h\|_{q}^{q} \\
\|h\|_{q}^{q} & =\int_{M} h^{q} d \mu=q \int_{M}\left(\int_{0}^{h(x)} u^{q-1} d u\right) d \mu_{x} \\
& =q \int_{M} d \mu_{x} \int_{0}^{\infty} d u u^{q-1} \Theta(h(x)-u),
\end{aligned}
$$

where $\Theta$ is the Heaviside function $\Theta(x)=1$ for $x \geq 0$ and $\Theta(x)=0$ otherwise. The integrand in (4.7) is positive, and thus we may commute the integrals according to Fubini's theorem,

$$
\begin{aligned}
\|h\|_{q}^{q} & =q \int_{0}^{\infty} d u u^{q-1} \int_{M} \Theta(h(x)-u) d \mu_{x} \\
& =q \int_{0}^{\infty} u^{q-1} V_{u} d u=q \int_{0}^{\infty} u^{q-1} V_{u}^{\frac{1}{n}} V_{u}^{\frac{n-1}{n}} d u .
\end{aligned}
$$

The isoperimetric inequality bounds the factor $V_{u}^{\frac{n-1}{n}}$ from above by $A_{u} / k$. The factor $V^{\frac{1}{n}}$, on the other hand, can be estimated with (4.6). We thus obtain the inequality

$$
\|h\|_{q}^{q} \leq \frac{q}{k}\|h\|_{q}^{\frac{q}{n}} \int_{0}^{\infty} u^{\frac{q}{2}} A_{u} d u .
$$

We finally substitute in (4.5) and apply the Schwarz inequality,

$$
\|h\|_{q}^{q} \leq \frac{q}{k}\|h\|_{q}^{\frac{q}{n}} \int_{M} h^{\frac{q}{2}}|\nabla h| d \mu \leq \frac{q}{k}\|h\|_{q}^{q-1}\|\nabla h\|_{2} .
$$

\section{Proof of the Curvature Estimates.}

We derive a pointwise estimate of the curvature tensor in terms of the spinors $\psi^{i}$ and their second derivatives. 


\section{Lemma 5.1.}

$$
\left(N-\sqrt{8 N}\left\|\mathbb{1}-P_{x}\right\|\right)|R|^{2} \leq 32 \sum_{i=1}^{N}\left|\nabla^{2} \psi^{i}\right|^{2} .
$$

Proof: For convenience we again choose an orthonormal frame $s_{1}, \ldots, s_{n}$ with $\nabla s_{\alpha}(x)=0$. The definition of curvature (2.1) and the Schwarz inequality give the following relation between curvature and the second derivatives of $\psi$,

$$
\begin{aligned}
& -\sum_{\alpha, \beta=1}^{n}\left\langle\psi, R^{S}\left(s_{\alpha}, s_{\beta}\right)^{2} \psi\right\rangle=\sum_{\alpha, \beta=1}^{n}\left\langle R^{S}\left(s_{\alpha}, s_{\beta}\right) \psi, R^{S}\left(s_{\alpha}, s_{\beta}\right) \psi\right\rangle \\
& =\sum_{\alpha, \beta=1}^{n}\left\langle\nabla_{s_{\alpha}, s_{\beta}}^{2} \psi-\nabla_{s_{\beta}, s_{\alpha}}^{2} \psi, \nabla_{s_{\alpha}, s_{\beta}}^{2} \psi-\nabla_{s_{\beta}, s_{\alpha}}^{2} \psi\right\rangle \\
& =\sum_{\alpha, \beta=1}^{n}\left(\left|\nabla_{s_{\alpha}, s_{\beta}}^{2} \psi\right|^{2}+\left|\nabla_{s_{\beta}, s_{\alpha}}^{2} \psi\right|^{2}-2\left\langle\nabla_{s_{\alpha}, s_{\beta}}^{2} \psi, \nabla_{s_{\beta}, s_{\alpha}}^{2} \psi\right\rangle\right. \\
& \leq 2 \sum_{\alpha, \beta=1}^{n}\left(\left|\nabla_{s_{\alpha}, s_{\beta}}^{2} \psi\right|^{2}+\left|\nabla_{s_{\beta}, s_{\alpha}}^{2} \psi\right|^{2}\right)=4\left|\nabla^{2} \psi\right|
\end{aligned}
$$

Using (2.2) and (2.3), the square of spinor curvature is computed to be

$$
\begin{aligned}
& \sum_{\alpha, \beta=1}^{n} R^{S}\left(s_{\alpha}, s_{\beta}\right)^{2} \\
& =\frac{1}{16} \sum_{\alpha, \beta=1}^{n} \sum_{\gamma, \delta, \varepsilon, \rho=1}^{n} R\left(s_{\gamma}, s_{\delta}, s_{\alpha}, s_{\beta}\right) R\left(s_{\varepsilon}, s_{\rho}, s_{\alpha}, s_{\beta}\right) s_{\gamma} s_{\delta} s_{\varepsilon} s_{\rho}
\end{aligned}
$$

$$
=-\frac{1}{8}|R|^{2}+\frac{1}{16} \sum_{\alpha, \beta=1}^{n} \sum_{\gamma, \delta, \varepsilon, \rho \text { all different }} R\left(s_{\gamma}, s_{\delta}, s_{\alpha}, s_{\beta}\right) R\left(s_{\varepsilon}, s_{\rho}, s_{\alpha}, s_{\beta}\right) s_{\gamma} s_{\delta} s_{\varepsilon} s_{\rho} .
$$

In dimension $n \leq 3$, the second term in (5.2) clearly vanishes, and so (5.2) is a multiple of the identity matrix, making it possible to proceed as in [4]. In order to control the second term in (5.2), we consider the expectation value 
with respect to all $\psi^{i}$ s and take their sum,

$$
\begin{gathered}
-\sum_{i=1}^{N} \sum_{\alpha, \beta=1}^{n}\left\langle\psi^{i}, R^{S}\left(s_{\alpha}, s_{\beta}\right)^{2} \psi^{i}\right\rangle=-\sum_{\alpha, \beta=1}^{n} \operatorname{Tr}\left(R^{S}\left(s_{\alpha}, s_{\beta}\right)^{2} P_{x}\right) \\
=-\sum_{\alpha, \beta=1}^{n} \operatorname{Tr}\left(R^{S}\left(s_{\alpha}, s_{\beta}\right)^{2}\right)+\sum_{\alpha, \beta=1}^{n} \operatorname{Tr}\left(R^{S}\left(s_{\alpha}, s_{\beta}\right)^{2}\left(\mathbb{1}-P_{x}\right)\right) \\
\geq-\sum_{\alpha, \beta=1}^{n} \operatorname{Tr}\left(R^{S}\left(s_{\alpha}, s_{\beta}\right)^{2}\right)-\left\|\sum_{\alpha, \beta=1}^{n} R^{S}\left(s_{\alpha}, s_{\beta}\right)^{2}\right\|\left\|\mathbb{1}-P_{x}\right\|
\end{gathered}
$$

A straightforward calculation using (5.2) shows that

$$
-\sum_{\alpha, \beta=1}^{n} \operatorname{Tr}\left(R^{S}\left(s_{\alpha}, s_{\beta}\right)^{2}\right)=\frac{N}{8}|R|^{2}, \quad\left\|\sum_{\alpha, \beta=1}^{n} R^{S}\left(s_{\alpha}, s_{\beta}\right)^{2}\right\| \leq \sqrt{\frac{N}{8}}|R|^{2} .
$$

Substituting these formulas into (5.3) and using (5.1) gives the result.

Our main result follows by combining Lemma 4.2, Corollary 3.2, and Lemma 5.1.

Proof of Theorem 1.2: We choose the set $D$ as in Lemma 4.2 corresponding to $\varepsilon=N / 32$. Then according to (4.2) and Lemma 5.1,

$$
\int_{M \backslash D} \eta|R|^{2} d \mu \leq \int_{M \backslash D} \eta \frac{64}{N} \sum_{i=1}^{N}\left|\nabla^{2} \psi^{i}\right|^{2} d \mu \leq \frac{64}{N} \sum_{i=1}^{N} \int_{M} \eta\left|\nabla^{2} \psi^{i}\right|^{2} d \mu,
$$

where in the last step we used the positivity of the integrand. Now apply Corollary 3.2 .

Acknowledgments: We would like to thank Robert Bartnik and Helga Baum for helpful suggestions and comments. We are grateful to Hubert Bray for many inspiring discussions and valuable ideas.

\section{References.}

[1] Schoen, R., Yau, S.-T., "On the proof of the positive mass conjecture in General Relativity," Commun. Math. Phys. 65 (1976) 45-76

[2] Witten, E., "A new proof of the positive energy theorem," Commun. Math. Phys. 80 (1981) 381-402 
[3] Bartnik, R., "The mass of an asymptotically flat manifold," Comm. Pure Appl. Math. 39 (1986) 661-693

[4] Bray, H., Finster, F., "Curvature estimates and the positive mass theorem," math.DG/9906047, Comm. Anal. Geom. 10 (2002) 291-306

[5] Parker, T., Taubes, C. H., "On Witten's proof of the positive energy theorem," Commun. Math. Phys. 84 (1982) 223-238

[6] Baum, H., "Spin-Strukturen und Dirac-Operatoren über pseudoriemannschen Mannigfaltigkeiten," Teubner Verlag Leipzig (1981)

[7] Lawson, H.B., Michelson M.-L., "Spin Geometry," Princeton Univ. Press (1989)

NWF I - MATHEMATIK

UNIVERSITÄT REGENSBURG

93040 Regensburg, GeRmany

E-mail: Felix.Finster@mathematik. uni-regensburg.de

Max Planck Institute for Mathematics in the Sciences

INSELSTR. 22-26, 04103 LEIPZIG, GERMANY

E-mail: ikath@mis.mpg.de 